International Journal of Pure and Applied Mathematics

Volume 114 No. 2 2017, 407-418

ISSN: 1311-8080 (printed version); ISSN: 1314-3395 (on-line version)

url: http://www.ijpam.eu

doi: 10.12732 /jpam.v114i2.20

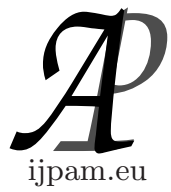

\title{
TWO-DIMENSIONAL PROBLEM CONCERNING CONTACT SUFFUSION INTERPLAY ON THE FILTRATION CONSOLIDATION PROCESSES OF HETEROGENEOUS SOILS
}

\author{
Volodymyr Herus ${ }^{1}$, Petro Martyniuk ${ }^{2}$, \\ Olha Michuta ${ }^{3}$, Olha Stepanchenko ${ }^{4}$ \\ 1,2,3,4 National University of Water and \\ Environmental Engineering \\ Rivne, UKRAINE
}

\begin{abstract}
An improved mathematical model of the interrelated filtration consolidation processes, heat and mass transfer as well as contact suffusion in heterogeneous soils has been built and researched. A solution of the boundary problem has been programmed with the finite elements method. The influence of the suffusion phenomena on the soil surface subsidence has been analyzed based on a model problem.
\end{abstract}

AMS Subject Classification: $65 \mathrm{C} 20$

Key Words: finite elements method, soil consolidation, mechanical suffusion, heat and mass transfer

\section{Introduction}

For the civil as well as industrial building, the questions of uneven soil deformations under civil and industrial buildings remain topical. These subsidence processes are conditioned by a bundle of factors, that are connected with the human activity (e.g. the heat and chemical regime change $[1,2]$ ), as well as with some environmental of the region (e.g. gypsum containing soils [3].

Received: February 21, 2017

Revised: $\quad$ May 5, 2017

Published: $\quad$ May 8, 2017

(C) 2017 Academic Publications, Ltd. url: www.acadpubl.eu

$\S_{\text {Correspondence author }}$ 
Starting from the 30 s of the XX century together with the transfer to a strict mathematical basis for the scientific researches in the soil mechanics field has allowed bringing a number of problematic questions to the mathematical modelling, as well as to the equation theory of the mathematical physics and numerical methods, used to solve the appropriate boundary problems. It is not a coincidence that in the boundary problems theory of the mathematical physics, in the areas with agile boundaries together with the classical Stephan's problem there also is the so-called Florin's problem [4]. This problem stems from the soils consolidation (sealing) problem under the influence of some external burden or the soils own mass [5]. As it turned out (taking into consideration certain assumptions), the problem of a fully saturated soil consolidation can be brought to the problem of excessive pressures in a porous liquid, as well as their dispersion levels determination. Therefore, from the mathematical physics standpoint, its mathematical model is described by the boundary problem for an equation (in a classical case - of the parabolic type), where the unknown function is the excessive pressures one. The excessive pressures decrease (or increase) grade influences the soil surface flash of swelling. This is why the consolidation problem, in a general case, is a problem with moving boundaries $[6]$.

In the works $[1,2]$ a theory of filtration consolidation has been developed with an account on a technogenic influence as well as the filtration soil destruction. The thermo- salt transfer phenomenon, as well as the phenomena of the chemical suffusion and the presence of the soil wash-out zones, cleated by a filtration flow have been taken into consideration. Mathematical models of the examined problems are described by the boundary problems for the nonlinear partial differential equation systems in the areas with moving boundaries. The elements of a quality theory of such one-dimensional boundary problems can be found in [6] (see also the applied bibliography).

Still, except for the contact washout processes, the contact suffusion phenomena also take place in heterogeneous soils. If two types of soils (one of a smaller fraction, the other - of a bigger one) have common border, and the filtration speed by the transition through the border exceeds the critical value, then the smaller fraction particles can mix into the pores of the bigger fraction soil. As a result - the border between the two soils is mobile, which can influence the soil body subsidence [7]. This phenomenon (of the contact suffusion) is important during the construction of reverse filters in soil dams [8]. As the increased pressures, which result in the increased filtration speeds, accompany the consolidation processes, a topical problem of the connected consolidation and suffusion processes arises. This article's aim is to research the two-dimensional 


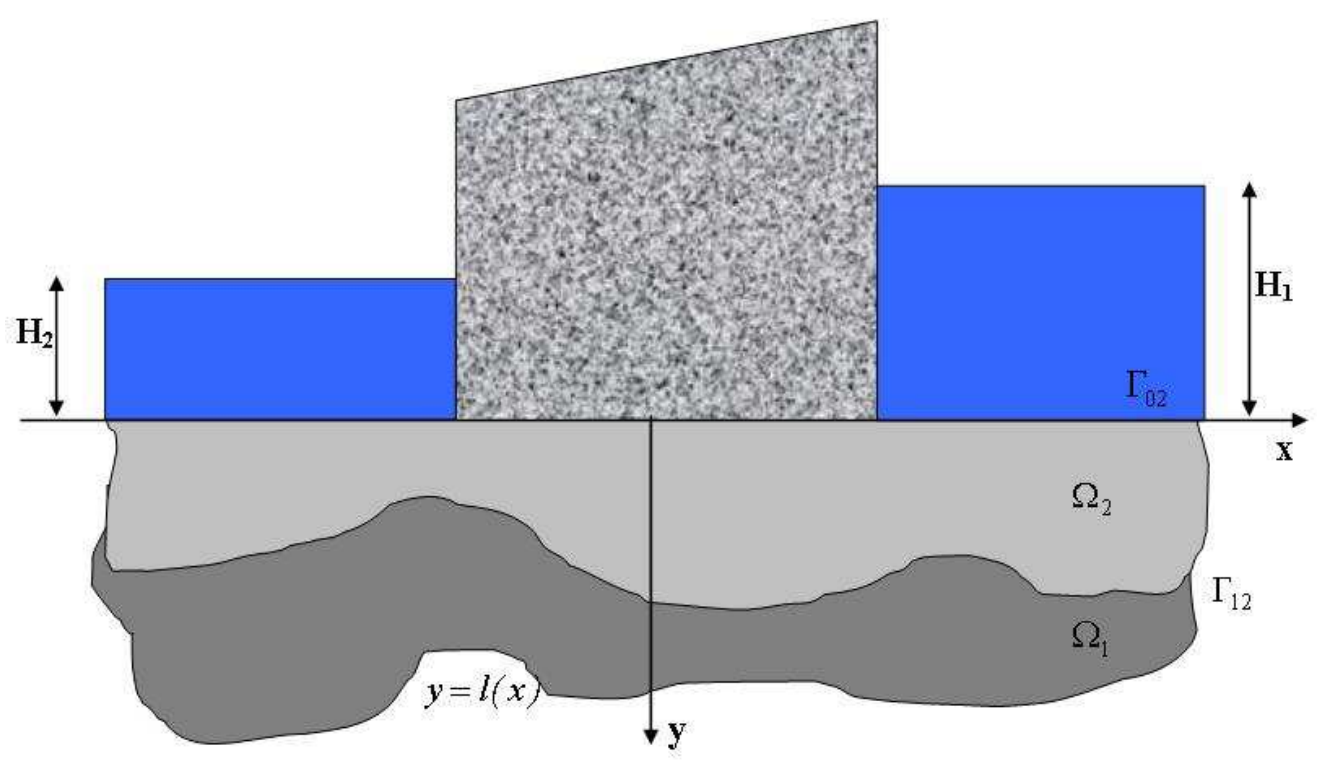

Figure 1: Filtration consolidation and contact suffusion in a heterogeneous soil body

problem under the thermal and solute transfer conditions.

\section{The Problem Mathematical Model and Statement}

Let us examine a soil body in the area $\Omega_{1}$, which is laden by the soil of a bigger fraction in the area $\Omega_{2}$ (figure 1). Under the influence of an ascending filtration flow the suffusion particles from the area $\Omega_{1}$ can penetrate the area $\Omega_{2}$. As a result, the contact border between the two soils $y=\Gamma_{12}(x, t)$ is mobile and changes its place in time. Therefore, in the area $\Omega_{2}$ the soil is located, that consists out of a "skeleton", and suffusion particles in number of $s$ fractions with concentrations $\sigma^{(i)}(X, t), i=\overline{1, s}$ (here $\left.X=(x, y)\right)$. Under the concentration of the $\sigma^{(i)}(X, t), i=\overline{1, s}$ particles in a certain separated volume $\mathrm{V}$ of a porous environment shall be seen the correlation of the suffusion particles volume to the whole separated volume $\mathrm{V}$. That is why $\sigma_{\max }(X, t)=\left(1-n_{1}\right) \cdot n_{2}, n_{i}$ functions of the soil's porosity on the areas $\Omega_{i}, i=1,2$ (where $n_{2}$ - is the porosity without taking into consideration the presence of mobile suffusion particles).

In order to avoid any misunderstandings the following should be highlighted. The upper mobile border as well as the two bodies contact border are described 
by the equations in their explicit form $y=\Gamma_{02}(x, t), y=\Gamma_{12}(x, t)$ accordingly. Later the implicit forms of the equations $\Gamma_{02}(X, t)=0, \Gamma_{12}(X, t)=0$ where $\Gamma_{02}(X, t)=y-\Gamma_{02}(x, t), \Gamma_{12}(X, t)=y-\Gamma_{12}(x, t)$ will also be used. In order to avoid extra markings the same " $\Gamma_{02}$ " and " $\Gamma_{12}$ " were used, which differ with arguments. The lower immobile border is described with an equation $y=l(x)$.

The mathematical model of the filtration consolidation problem with an account for multifractional suffusion according to the above given statement is described by the following boundary value problem:

$$
\begin{gathered}
\frac{\partial h(X, t)}{\partial t}=\frac{(1+e)(1+\xi)}{2 \gamma a\left(1-\sum_{i=1}^{s} \sigma^{(i)}\right)} \times \\
\times \nabla \cdot\left(K_{h}(c, T, \sigma) \nabla h(X, t)-K_{c} \nabla c(X, t)-K_{T} \nabla T(X, t)\right), \\
\nabla \cdot(D \nabla c(X, t))+\nabla \cdot\left(D_{T} \nabla T(X, t)\right)-u \nabla c(X, t)=n \frac{\partial c}{\partial t}+\frac{\partial N}{\partial t}, \\
\nabla \cdot(\lambda \nabla T(X, t))-\rho c_{\rho} \nabla T(X, t)=c_{T} \frac{\partial T}{\partial t}, \\
\nabla \cdot\left(D^{(i)} \nabla \sigma^{(i)}(X, t)-f\left(\sigma, \sigma_{\max }, u, u_{k r}^{(i)}\right) \cdot \sigma^{(i)}(X, t)\right)= \\
=\frac{\partial \sigma^{(i)}(X, t)}{\partial t}, i=\overline{1, s}, X \in \bar{\Omega}_{2}, \\
\frac{\partial N}{\partial t}=\gamma_{1}\left(c-C_{m}\right), \\
q^{(i)}(X, t)=-D^{(i)} \nabla \sigma^{(i)}(X, t)+ \\
+f\left(\sigma, \sigma_{\max }, u, u_{k r}^{(i)}\right) \cdot \sigma^{(i)}(X, t), i=\overline{1, s}, X \in \bar{\Omega}_{2}, \\
\left(\sigma_{\max }-\sum_{i=1}^{S} \sigma^{(i)}(X, t)\right) \frac{d \Gamma_{12}(x, t)}{d t}= \\
u=-K_{h}(c, T, \sigma) \nabla h(X, t)+K_{c} \nabla c(X, t)+K_{T} \nabla T(X, t),
\end{gathered}
$$




$$
\begin{gathered}
=-\sum_{i=1}^{S}\left(q^{(i)}, n_{12}\right),(X, t) \in \Gamma_{12}(X, t) \\
\frac{d \Gamma_{02}(x, t)}{d t}=-\frac{1}{n_{y}^{(12)}} \cdot \frac{d \Gamma_{12}(x, t)}{d t}+ \\
+\frac{2 \gamma a}{(1+e)(1+\xi)} \int_{\Gamma_{02}(x, t)}^{l(x)} \frac{\partial h(x, y, t)}{\partial t} d y,(X, t) \in \Gamma_{02}(X, t), \\
h(X, 0)=H_{0}(X), c(X, 0)=c_{0}(X), \\
T(X, 0)=T_{0}(X), \sigma_{m}^{(i)}(X, 0)=\sigma_{m_{\mathrm{i}}}^{(0)}(X), i=\overline{1, s} \\
\Gamma_{12}(X, 0)=\Gamma_{12}^{(0)}(X), \Gamma_{02}(X, 0)=\Gamma_{02}^{(0)}(X) .
\end{gathered}
$$

The boundary conditions are given in an abstract form. They depend on the problem statement specification

$$
\begin{gathered}
\left.h(X, t)\right|_{\Gamma_{02}}=H_{1}(X, t),\left.(u, n)\right|_{l}=0, \\
\left.c(X, t)\right|_{\Gamma_{02}}=c_{1}(X, t),\left.\left(q_{c}, n\right)\right|_{l}=0, \\
\left.T(X, t)\right|_{\Gamma_{02}}=T_{1}(X, t),\left.\left(q_{T}, n\right)\right|_{l}=0, \\
\left.\sigma^{(i)}(X, t)\right|_{x=\Gamma_{12}}=\sigma_{1}^{(i)}(X, t), i=\overline{1, s .}
\end{gathered}
$$

The conjugation conditions must be given on the contact border $y=\Gamma_{12}(x, t)$. Let us suppose, that the ideal contact condition is carried out on the border $y=\Gamma_{12}(x, t)$. Then:

$$
\begin{gathered}
{\left.[h(X, t)]\right|_{\Gamma_{12}}=0,\left.[(u, n)]\right|_{\Gamma_{12}}=0,} \\
{\left.[c(X, t)]\right|_{\Gamma_{12}}=0,\left.\left[\left(q_{c}, n\right)\right]\right|_{\Gamma_{12}}=0,} \\
{\left.[T(X, t)]\right|_{\Gamma_{12}}=0,\left.\left[\left(q_{T}, n\right)\right]\right|_{\Gamma_{12}}=0,}
\end{gathered}
$$


where $[\cdot]$ - function jump symbol.

The following markings were used in problem (1)-(19):

$h(X, t)$ - excessive pressure in pore liquid; $c(X, t)$ - salt solution concentration; $T(X, t)$ - temperature; $\sigma^{(i)}(X, t)$ - suffusion particles concentration of the $i$ fraction; $D^{(i)}$ - particles dispersion coefficient of the $i$-fraction; $a$ - soil compression coefficient; $D$ - convective diffusion coefficient; $D_{T}$ - thermal diffusion coefficient; $C_{m}$ - the boundary saturation concentration; $u(X, t)-$ filtration speed; $\nu$ - hard soil particles movement speed; $n$ - soil porosity; $K_{h}(c, T, e)-$ filtration coefficient; $K_{c}$ - chemical osmosis coefficient; $K_{T}$ - thermal osmosis coefficient; $c_{T}$ - soil voluminous heat capacity; $c_{\rho}$ - porous soil specific heat capacity; $\rho$ - porous solution density; $\lambda$ - soil thermal conductivity coefficient; $\gamma-$ porous fluid specific weight; $e$ - void ratio of soil; $f\left(\sigma, \sigma_{\max }, u, u_{k r}\right)=\left\{f_{j}\right\}_{j=1}^{2}$, $f_{j}=\alpha_{e r}\left(u_{j}-u_{k r}\right)_{+}$, where $g_{+}=\left\{\begin{array}{l}g, g \geq 0 ; \\ 0, g<0 ;\end{array}, \alpha_{e r}\right.$ - wash-out coefficient; $u_{k r}$ - wash-out scalar critical speed, the value for which can differ for different fractions.

As the whole soil body is heterogeneous on the macro level, therefore, for example $n=\left\{n_{i}, X \in \Omega_{i},(i=1,2)\right\}$. This applies for the other coefficients and unknown functions of the problem (1)-(19).

As seen from (4) and (6) the diffusion model $[9,10]$ was chosen as a suffusion distribution model.

\section{The Numerical Solution of the Boundary Problem by the Finite Element Method}

The numerical solution of the problem (1)-(19) will be found using the finite element method (FEM), using the discontinuous functions [11]. Let us assume (without loss of generality) that the suffusion particles have only one fraction. That means $-s=1$. Then the concentration shall be marked $\sigma(X, t)$.

Each of the areas $\Omega_{1}$ and $\Omega_{2}$ will be covered with finite element meshes. It is important that on the border $\Gamma_{12}$ the initial knots' whereabouts to on each area's side $\Omega_{i},(i=1,2)$ have to be congruent. For a more detailed information on the rules and requirements for the finite element mesh in case of the problems in heterogeneous areas see [11]. A generalized solution of the boundary problem (1)-(19) will be find for in the following combination:

$$
T(X, t) \approx \tilde{T}(X, t)=\sum_{j=1}^{N} a_{j}(t) \cdot \varphi_{j}(X),
$$




$$
\begin{aligned}
& c(X, t) \approx \tilde{c}(X, t)=\sum_{j=1}^{N} b_{j}(t) \cdot \varphi_{j}(X), \\
& h(X, t) \approx \tilde{h}(X, t)=\sum_{j=1}^{N} c_{j}(t) \cdot \varphi_{j}(X), \\
& \sigma(X, t) \approx \tilde{\sigma}(X, t)=\sum_{j=1}^{N} s_{j}(t) \cdot \varphi_{j}(X),
\end{aligned}
$$

where $\varphi_{j}(X)$ - base FEM functions, that are discontonuous on the border $\Gamma_{12}$.

As the unknown coefficients in (20) depend on time only, then in accordance with the Galorkin method a Cauchy problem for the system of simple nonlinear differential equations is received:

$$
\begin{gathered}
M^{(1)} \cdot \frac{d A}{d t}+L^{(1)} \cdot A(t)=F^{(1)}, \\
M^{(1)} \cdot A(0)=P^{(1)}, \\
M^{(2)} \cdot \frac{d B}{d t}+L^{(2)} \cdot B(t)=G^{(1)} \cdot A(t)+F^{(2)}, \\
M^{(2)} \cdot B(0)=P^{(2)}, \\
\frac{d C}{d t}+L^{(3)} \cdot C(t)=G^{(2)} \cdot A(t)+G^{(3)} \cdot B(t)+F^{(3)}, \\
M^{(3)} \cdot C(0)=P^{(3)}, \\
M^{(4)} \cdot \frac{d S}{d t}+L^{(4)} \cdot S(t)=F^{(4)}, \\
M^{(4)} \cdot S(0)=P^{(4)},
\end{gathered}
$$

where

$$
\begin{gathered}
A=\left\{a_{j}\right\}_{j=1}^{N}, A(0)=\{a(0)\}_{j=1}^{N}, B=\left\{b_{j}\right\}_{j=1}^{N}, B(0)=\{b(0)\}_{j=1}^{N}, C= \\
\left\{c_{j}\right\}_{j=1}^{N}, C(0)=\{c(0)\}_{j=1}^{N}, S=\left\{s_{j}\right\}_{j=1}^{N}, S(0)=\{s(0)\}_{j=1}^{N}, M^{(\xi)}=\left\{m_{i j}^{(\xi)}\right\}_{i=1, j=1}^{N, N},
\end{gathered}
$$


$L^{(\xi)}=\left\{l_{i j}^{(\xi)}\right\}_{i=1, j=1}^{N, N}, P^{(\xi)}=\left\{p_{i}^{(\xi)}\right\}_{i=1}^{N}, F^{(\xi)}=\left\{f_{i}^{(\xi)}\right\}_{i=1}^{N}, \xi=\overline{1,4}, G^{(\xi)}=$ $\left\{g_{i j}^{(\xi)}\right\}_{i=1, j=1}^{N, N}, \xi=\overline{1,3}$.

For the matrixes $M^{(i)}, L^{(i)}, i=\overline{1,4}, G^{(i)}, i=\overline{1,3}$ and vector columns $F^{(i)}$, $P^{(i)}, i=\overline{1,4}$ we get the following representations on an arbitrary element $\mathrm{E}$ :

$$
\begin{aligned}
& m_{i j}^{(1)}=\sum_{E} \int_{\Omega} \int c_{T} \varphi_{j} \varphi_{i} d X ; m_{i j}^{(2)}=\sum_{E} \int_{\Omega} \int n \varphi_{j} \varphi_{i} d X \\
& l_{i j}^{(1)}=\sum_{E} \int_{\Omega} \int\left(\lambda(c) \nabla \varphi_{j} \nabla \varphi_{i}+\rho c_{\rho} u \cdot \nabla \varphi_{j} \varphi_{i}\right) d X ; \\
& l_{i j}^{(2)}=\sum_{E} \int_{\Omega} \int\left(D \nabla \varphi_{j} \nabla \varphi_{i}+u \cdot \nabla \varphi_{j} \varphi_{i}-\gamma_{1} \varphi_{j} \varphi_{i}\right) d X ; \\
& m_{i j}^{(3)}=\sum_{E} 2 \gamma a(1-\sigma) \cdot \int_{\Omega} \int \varphi_{j} \varphi_{i} d X ; m_{i j}^{(4)}=\sum_{E} \int_{\Omega} \int \varphi_{j} \varphi_{i} d X ; \\
& l_{i j}^{(3)}=\sum_{E} \int_{\Omega} \int(1+e)(1+\xi) K_{h} \nabla \varphi_{j} \nabla \varphi_{i} d X ; g_{i j}^{(1)}=\sum_{E} \int_{\Omega} \int D_{T} \nabla \varphi_{j} \nabla \varphi_{i} d X ; \\
& g_{i j}^{(2)}=\sum_{E} \int_{\Omega} \int K_{T} \nabla \varphi_{j} \nabla \varphi_{i} d X ; g_{i j}^{(3)}=\sum_{E} \int_{\Omega} \int K_{c} \nabla \varphi_{j} \nabla \varphi_{i} d X \\
& l_{i j}^{(4)}=\sum_{E} \int_{\Omega} \int\left(D_{s} \nabla \varphi_{j} \nabla \varphi_{i}+f \cdot \nabla \varphi_{j} \varphi_{i}\right) d X ; \\
& p_{i}^{(1)}=\sum_{E} \int_{\Omega} \int T_{0} \varphi_{i} d X ; p_{i}^{(2)}=\sum_{E} \int_{\Omega} \int C_{0} \varphi_{i} d X ; \\
& p_{i}^{(3)}=\sum_{E} \int_{\Omega} \int H_{0} \varphi_{i} d X ; p_{i}^{(4)}=\sum_{E} \int_{\Omega} \int \sigma^{(0)} \varphi_{i} d X ; \\
& f_{i}^{(2)}=\sum_{E} \int_{\Omega} \int \gamma_{1} C_{m} \varphi_{i} d X .
\end{aligned}
$$

Here the sums are taken from those finite elements $E$, which have knots with numbers $i$ and $j$ (as far as the vector columns are concerned - only number 
i). By the problem derivation in a weak form, only natural conditions were taken into consideration. The main conditions (17)-(19) have to be taken into consideration directly. The according scheme is described, e.g. in [11].

In order to find a numerical solution to the Cauchy problem (21)-(28) we shall cover the time space $\left[0, t_{0}\right]$ with a net $t_{j}=j \tau, j=\overline{0, k+1}$ with step $\tau=\frac{t_{0}}{m}$. Then, using an implicit difference scheme we get

$$
\begin{gathered}
\left(M^{(1)}+\tau \cdot L^{(1)(j)}\right) \cdot A^{(j+1)}=M^{(1)} \cdot A^{(j)}+\cdot \tau \cdot F^{(1)(j+1)}, \\
\left(M^{(2)}+\tau \cdot L^{(2)(j)}\right) \cdot B^{(j+1)}=\tau \cdot G^{(2)(j)} \cdot A^{(j+1)}+M^{(2)} \cdot B^{(j)}+\cdot \tau \cdot F^{(2)(j+1)}, \\
\left(M^{(3)}+\tau \cdot L^{(3)(j)}\right) \cdot C^{(j+1)}= \\
\tau \cdot G^{(2)(j)} \cdot A^{(j+1)}+G^{(3)(j)} \cdot B^{(j)}+M^{(2)} \cdot C^{(j)}+\tau \cdot F^{(3)(j+1)}, \\
\left(M^{(4)}+\tau \cdot L^{(4)(j)}\right) \cdot S^{(j+1)}=M^{(4)} \cdot S^{(j)}+\cdot \tau \cdot F^{(4)(j+1)} .
\end{gathered}
$$

The system of linear equations (29)-(32), received after a time discretization can be solved using a modified Gauss method.

As a result of kinematic boundary problem discretization (8) we get

$$
\left.\left(\sigma_{\max }-\sigma^{(j+1)}\right)\right|_{\Gamma_{12}^{(j)}} \cdot \frac{\Gamma_{12}^{(j+1)}-\Gamma_{12}^{(j)}}{\tau}=-\left(q^{(j+1)}, n_{12}\right),(j=\overline{1, k}) .
$$

From the equation (33) the location of the points on the contact border are found

$$
\Gamma_{12}^{(j+1)}=\frac{\tau}{\sigma_{\max }-\sigma^{(j+1)}} \cdot\left(q^{(j+1)}, n_{12}\right)+\Gamma_{12}^{(j)} .
$$

During the consolidation process the size of $\Omega$ are change. Therefore, the knot points coordinates are to be recalculated on every time step. To do this, the kinematic boundary condition (9) is used:

$$
\frac{\Gamma_{02}^{(j+1)}-\Gamma_{02}^{(j)}}{\tau}=-\frac{1}{n_{y}^{(j+1)}} \frac{\Gamma_{12}^{(j+1)}-\Gamma_{12}^{(j)}}{\tau}+
$$




$$
+\frac{2 a \gamma}{(1+e)(1+\xi)} \int_{\Gamma_{02}^{(j+1)}}^{l} \frac{h^{(j+1)}-h^{(j)}}{\tau} d y,(j=\overline{0, k-1}) .
$$

From the equation (34) the location of the upper mobile soil border on the time step $(j+1)$ is distinguished. The integral on the right side (34) can be calculated, using quadrature formulas of Gauss. The integration variable $y$ passes a vertical segment from the knot's starting point $y^{(j)}$ all the way until the soil body lower border 1 .

\section{Numeric Experiments' Results and Their Analysis}

Let us examine a soil body of a rectangular shape with an initial thickness of $15 \mathrm{~m}$, and a width of $10 \mathrm{~m}$. The soil layer separation is located on the depth of $8 \mathrm{~m}$. Therefore, the initial equation of $y=\Gamma_{12}(x, t)$ is $y=8$. Suppose that the lower soil border is impenetrable. An immediate outer load $q=10 \cdot 10^{4} \frac{\mathrm{kg}}{\mathrm{m}^{2} \cdot \mathrm{day}^{2}}$ is applied evenly to the upper soil border. The other values follow:

$\gamma=10^{4} \mathrm{~Pa} / \mathrm{m}^{3}, a_{1}=10^{-7} \mathrm{~m}^{2}, a_{2}=10^{-6} \mathrm{~m}^{2}, e_{1}=0.62, e_{2}=0.5, \sigma_{0}(X)=$ $0, C_{m}=100 \mathrm{~kg} / \mathrm{m}^{3}, H_{1}(X, t)=0 \mathrm{~m}, H_{0}(X)=10 \mathrm{~m}, u_{k r}=0 \mathrm{~m} / \mathrm{day}$, $K_{T}^{(1,2)}=2.8 \cdot 10^{-6} \mathrm{~m}^{2} /{ }^{o} \mathrm{C} \cdot \mathrm{day}, \rho=1100 \mathrm{~kg} / \mathrm{m}^{3}, c_{T}^{(1,2)}=2.1 \cdot 10^{6} \mathrm{~J} /{ }^{\circ} \mathrm{C} \cdot \mathrm{m}^{3}$, $K_{c}^{(1,2)}=2.8 \cdot 10^{-5} \mathrm{~m}^{-5} / \mathrm{kg} \cdot \operatorname{day}, \lambda^{(1,2)}=108 \cdot 10^{3} \mathrm{~J} /{ }^{o} \mathrm{C} \cdot \mathrm{m} \cdot \mathrm{day}, C_{0}(X)=$ $\left\{\begin{array}{l}C_{m}, x=0 \\ 5 \mathrm{~kg} / \mathrm{m}^{3}, x \neq 0 ;\end{array} T_{0}(X)=\left\{\begin{array}{l}20^{\circ} C, x=0 \\ 4^{\circ} C, x \neq 0\end{array}\right.\right.$

Filtration coefficients in case of clear water and the absence of a thermal factor $-K_{h}^{1}=0.01 \mathrm{~m} / \mathrm{day}, K_{h}^{2}=0.1 \mathrm{~m} / \mathrm{day}$. In the general case the filtration coefficient depends on soil temperature and concentration of salt solution.

The number of finite elements - 414. The piecewise-linear base functions were used. The time of the consolidation process observation equals 360 days. The chosen time step - 30 days.

There have been a number of experiments, where heat and salt transfer, and filtration destruction interplay on the extra pressures change dynamics (see works $[1,2])$. Therefore, we concentrate on the influence of suffusion on the soil surface subsidence. The uneven soil subsidence is known to be the primary cause of the accidents, connected with the industrial and civil buildings. Such dynamics for this model problem for one knot is shown in the tables 1,2 . The results show that the relative subsidence due to the suffusion processes lies between $70 \%$ and $90 \%$. The processes dynamics is also substantially influenced by heat and salt transfer. In conclusion, there are model problems the main role in which play the processes of not just compression, but also of contact mechanical suffusion. 


\begin{tabular}{|l|l|l|l|}
\hline $\begin{array}{l}\text { Moment } \\
\text { of time, } \\
\text { day }\end{array}$ & $\begin{array}{l}\text { The overall } \\
\text { subsidence, } \\
\mathrm{m}\end{array}$ & $\begin{array}{l}\text { The subsidence } \\
\text { due to the suf- } \\
\text { fusion, } \mathrm{m}\end{array}$ & $\begin{array}{l}\text { of subsidence } \\
\text { as a result of } \\
\text { suffusion form } \\
\text { the overall } \\
\text { subsidence }\end{array}$ \\
\hline 60 & 0.043 & 0.0315 & 73.2852 \\
\hline 120 & 0.0623 & 0.0484 & 77.7664 \\
\hline 180 & 0.0579 & 0.0612 & 80.5999 \\
\hline 240 & 0.087 & 0.0718 & 82.5591 \\
\hline 300 & 0.0965 & 0.0811 & 84.0178 \\
\hline 360 & 0.1051 & 0.0895 & 85.1529 \\
\hline
\end{tabular}

Table 1: The presentage of subsidence due to the concentration of suffosion particles from the general knot subsidence $(0 ; 1,08)$ with time (heat and salt transfer were counted in)

\begin{tabular}{|l|l|l|l|}
\hline $\begin{array}{l}\text { Moment } \\
\text { of time, } \\
\text { day }\end{array}$ & $\begin{array}{l}\text { The overall } \\
\text { subsidence, } \\
\mathrm{m}\end{array}$ & $\begin{array}{l}\text { The subsidence } \\
\text { due to the suf- } \\
\text { fusion, m }\end{array}$ & $\begin{array}{l}\text { of subsidence } \\
\text { as a result of } \\
\text { suffusion form } \\
\text { the overall } \\
\text { subsidence }\end{array}$ \\
\hline 60 & 0.0359 & 0.0315 & 87.8183 \\
\hline 120 & 0.0558 & 0.0484 & 86.7762 \\
\hline 180 & 0.0703 & 0.0612 & 87.021 \\
\hline 240 & 0.082 & 0.0718 & 87.5127 \\
\hline 300 & 0.0922 & 0.0811 & 88.0242 \\
\hline 360 & 0.1011 & 0.0895 & 88.5017 \\
\hline
\end{tabular}

Table 2: The presentage of subsidence due to the suffosion from the general knot subsidence $(0 ; 1,08)$ with time (heat and salt transfer were not counted in)

\section{Conclusion}

A mathematical model of the interrelated processes of soil consolidation, contact suffusion, heat and salt transfer was researched numerically in this article. The test example shows the possibility of a considerable influence of the contact suffusion processes on the soil surface subsidence. The questions of the finite element solutions of the corresponding class of the nonlinear boundary problems 
accuracy, as well as the question of existence and uniqueness of their solutions were left out the authors' scope. The mentioned questions will be addressed in the authors' upcoming works in this direction.

\section{References}

[1] A. P. Vlasyuk, P. M. Martinyuk, O. R. Fursovych, Numerical solution of a onedimensional problem of filtration consolidation of saline soils in a nonisothermal regime, J. Mathematical Sciences, 160, 4 (2009), 525-535. doi: 10.1007/s10958-009-9518-8

[2] A. P. Vlasyuk, P. M. Martinyuk, Numerical solution of three-dimensional problems of filtration consolidation with regard for the influence of technogenic factors by the method of radial basis functions, J. Mathematical Sciences, 171, 5 (2010), 632-648. doi: 10.1007/s10958-010-0163-z

[3] V. P. Petrukhin, Calculation of suffosion deformations in saline soils, Soil Mechanics and Foundation Engineering, 32, 5 (1995), 159-162. doi: 10.1007/BF02336297

[4] G. I. Bizhanova, Classical solvability for one-dimensional, free-boundary, Florin, MuskatVerigin, and Stefan problems, J. Mathematical Sciences, 99, 1 (2000), 816-836. doi:10.1007/BF02673591

[5] Yu. K. Zaretskii, Theory of soil consolidation, IPST, Jerusalem (1972).

[6] P. M. Martinyuk, Existence and uniqueness of a solution of the problem with free boundary in the theory of filtration consolidation of soils with regard for the influence of technogenic factors, J. Mathematical Sciences, 207, 1 (2015), 59-73. doi: 10.1007/s10958-015$2355-\mathrm{z}$

[7] P. M. Martinyuk, O. V. Hoschko, Mathematical model of soils filtration consolidation taking into account multi-grade suffosion, Bulletin of Taras Shevchenko National University of Kyiv. Series: Physics 83 Mathematics, 4 (2013), 136-141 (in Ukrainian).

[8] A. K. Raut, Mathematical modeling of granular filters and constriction-based filter design criteria, PhD, Department of Civil Engineering, University of Wollongong (2006).

[9] C. V. Chrysikopoulos, V. E. Katzourakis, Colloid particle size-dependent dispersivity, Water Resources Research, 51, 6 (2015), 4668-4683. doi: 10.1002/2014WR016094

[10] A. P. Safonyk, Modelling the filtration processes of liquids from multicomponent contamination in the conditions of aythentication of mass transfer coefficient, Int. J. of Mathematical Models and Methods in Applied Sciences, 9 (2015), 189-192.

[11] I. V. Sergienko, V. V. Skopetskyi, V. S. Dineka, Mathematical modeling and research of processes in heterogeneous environments, Nauk.dumka, Kiev (1991) (in Russian). 